\title{
A Double-Parameter GPMHSS Method for a Class of Complex Symmetric Linear Systems from Helmholtz Equation
}

\author{
Cui-Xia Li and Shi-Liang Wu \\ School of Mathematics and Statistics, Anyang Normal University, Anyang 455000, China \\ Correspondence should be addressed to Shi-Liang Wu; wushiliang1999@126.com
}

Received 11 November 2013; Accepted 5 February 2014; Published 7 April 2014

Academic Editor: Masoud Hajarian

Copyright ( 92014 C.-X. Li and S.-L. Wu. This is an open access article distributed under the Creative Commons Attribution License, which permits unrestricted use, distribution, and reproduction in any medium, provided the original work is properly cited.

Based on the preconditioned MHSS (PMHSS) and generalized PMHSS (GPMHSS) methods, a double-parameter GPMHSS (DGPMHSS) method for solving a class of complex symmetric linear systems from Helmholtz equation is presented. A parameter region of the convergence for DGPMHSS method is provided. From practical point of view, we have analyzed and implemented inexact DGPMHSS (IDGPMHSS) iteration, which employs Krylov subspace methods as its inner processes. Numerical examples are reported to confirm the efficiency of the proposed methods.

\section{Introduction}

Let us consider the following form of the Helmholtz equation:

$$
-\Delta u+\sigma_{1} u+i \sigma_{2} u=f
$$

where $\sigma_{1}, \sigma_{2}$ are real coefficient functions and $u$ satisfies Dirichlet boundary conditions in $D=[0,1] \times[0,1]$.

Using the finite difference method to discretize the Helmholtz equation (1) with both $\sigma_{1}$ and $\sigma_{2}$ strictly positive leads to the following system of linear equations:

$$
A x=b, \quad A \in \mathbb{C}^{n \times n}, x, b \in \mathbb{C}^{n},
$$

where $A=W+i T \in \mathbb{C}^{n \times n}$ and $i=\sqrt{-1}$ with real symmetric matrices $W, T \in \mathbb{R}^{n \times n}$ satisfying $-W \preceq T \prec W$ under certain conditions. Throughout the paper, for $\forall B, C \in \mathbb{R}^{n \times n}, B \prec C$ means that $B-C$ is symmetric negative definite and $B \preceq C$ means that $B-C$ is symmetric negative semidefinite.

Systems such as (2) are important and arise in a variety of scientific and engineering applications, including structural dynamics [1-3], diffuse optical tomography [4], FFT-based solution of certain time-dependent PDEs [5], lattice quantum chromodynamics [6], molecular dynamics and fluid dynamics [7], quantum chemistry, and eddy current problem [8, 9]. One can see $[10,11]$ for more examples and additional references.
Based on the specific structure of the coefficient matrix $A$, one can verify that the Hermitian and skew-Hermitian parts of the complex symmetric matrix $A$, respectively, are

$$
H=\frac{1}{2}\left(A+A^{*}\right)=W, \quad S=\frac{1}{2}\left(A-A^{*}\right)=i T .
$$

Obviously, the above Hermitian and skew-Hermitian splitting (HSS) of the coefficient matrix $A$ is in line with the real and imaginary parts splitting of the coefficient matrix $A$. Based on the HSS method [12], Bai et al. [2] skillfully designed the modified HSS (MHSS) method to solve the complex symmetric linear system (2). To generalize the concept of this method and accelerate its convergence rate, Bai et al. in $[3,13]$ designed the preconditioned MHSS (PMHSS) method. It is noted that MHSS and PMHSS methods can efficiently solve the linear system (2) with $W>0$ and $T \geq 0$.

Recently, Xu [14] proposed the following GPMHSS method for solving the complex symmetric linear systems (2) with $-W \preceq T \prec W$ and it is described in the following.

The GPMHSS Method. Let $x^{(0)} \in \mathbb{C}^{n}$ be an arbitrary initial guess. For $k=0,1,2, \ldots$ until the sequence of iterates $\left\{x^{(k)}\right\}_{k=0}^{\infty}$ 
converges, compute the next iterate $x^{(k+1)}$ according to the following procedure:

$$
\begin{gathered}
(\alpha V+W-T) x^{(k+1 / 2)}=(\alpha V-i(W+T)) x^{(k)}+(1+i) b \\
(\alpha V+W+T) x^{(k+1)}=(\alpha V+i(W-T)) x^{(k+1 / 2)}+(1-i) b
\end{gathered}
$$

where $\alpha$ is a given positive constant and $V \in \mathbb{R}^{n \times n}$ is a prescribed symmetric positive definite matrix.

Theoretical analysis in [14] shows that the GPMHSS method converges unconditionally to the unique solution of the complex symmetric linear system (2). Numerical experiments are given to show the effectiveness of the GMHSS method.

In this paper, based on the asymmetric HSS and generalized preconditioned HSS methods in $[15,16]$, a natural generalization for the GMHSS iteration scheme is that we can design a double-parameter GMHSS (DGPMHSS) iteration scheme for solving the complex symmetric linear systems (2) with $-W \preceq T \prec W$. That is to say, the DGPMHSS iterative scheme works as follows.

The DGPMHSS Method. Let $x^{(0)} \in \mathbb{C}^{n}$ be an arbitrary initial guess. For $k=0,1,2, \ldots$ until the sequence of iterates $\left\{x^{(k)}\right\}_{k=0}^{\infty}$ converges, compute the next iterate $x^{(k+1)}$ according to the following procedure:

$$
\begin{aligned}
(\alpha V+W-T) x^{(k+1 / 2)}=( & \alpha V-i(W+T)) x^{(k)}+(1+i) b \\
(\beta V+W+T) x^{(k+1)}= & (\beta V+i(W-T)) x^{(k+1 / 2)} \\
& +(1-i) b
\end{aligned}
$$

where $\alpha$ is a given nonnegative constant, $\beta$ is a given positive constant, and $V \in \mathbb{R}^{n \times n}$ is a prescribed symmetric positive definite matrix.

Just like the GPMHSS method (4), both matrices $\alpha V+$ $W-T$ and $\beta V+W+T$ are symmetric positive definite. Hence, the two linear subsystems in (5) can also be effectively solved either exactly by a sparse Cholesky factorization or inexactly by a preconditioned conjugate gradient scheme. Theoretical analysis shows that the iterative sequence produced by DGPMHSS iteration method converges to the unique solution of the complex symmetric linear systems (2) for a loose restriction on the choices of $\alpha$ and $\beta$. The contraction factor of the DGPMHSS iteration can be bounded by a function, which is dependent only on the choices of $\alpha$ and $\beta$, the smallest eigenvalues of matrices $V^{-1}(W-T)$ and $V^{-1}(W+$ T).

This paper is organized as follows. In Section 2, we study the convergence properties of the DGPMHSS method. In Section 3, we discuss the implementation of DGPMHSS method and the corresponding inexact DGPMHSS (IDPGMHSS) iteration method. Numerical examples are reported to confirm the efficiency of the proposed methods in Section 4. Finally, we end the paper with concluding remarks in Section 5.

\section{Convergence Analysis for the DGPMHSS Method}

In this section, the convergence of the DGPMHSS method is studied. The DGPMHSS iteration method can be generalized to the two-step splitting iteration framework. The following lemma is required to study the convergence rate of the DGPMHSS method.

Lemma 1 (see [13]). Let $A \in \mathbb{C}^{n \times n}, A=M_{i}-N_{i}(i=1,2)$ be two splittings of $A$, and let $x^{(0)} \in \mathbb{C}^{n}$ be a given initial vector. If $\left\{x^{(k)}\right\}$ is a two-step iteration sequence defined by

$$
\begin{aligned}
& M_{1} x^{(k+1 / 2)}=N_{1} x^{(k)}+b, \\
& M_{2} x^{(k+1)}=N_{2} x^{(k+1 / 2)}+b,
\end{aligned}
$$

$k=0,1, \ldots$, then

$$
\begin{array}{r}
x^{(k+1)}=M_{2}^{-1} N_{2} M_{1}^{-1} N_{1} x^{(k)}+M_{2}^{-1}\left(I+N_{2} M_{1}^{-1}\right) b, \\
k=0,1, \ldots
\end{array}
$$

Moreover, if the spectral radius $\rho\left(M_{2}^{-1} N_{2} M_{1}^{-1} N_{1}\right)<1$, then the iterative sequence $\left\{x^{(k)}\right\}$ converges to the unique solution $x_{*} \in \mathbb{C}^{n}$ of system (1) for all initial vectors $x^{(0)} \in \mathbb{C}^{n}$.

Applying this lemma to the DGPMHSS method, we get convergence property in the following theorem.

Theorem 2. Let $\widehat{W}=R^{-T}(W-T) R^{-1}, \widehat{T}=R^{-T}(W+T) R^{-1}$, and $V=R^{T} R$, where $R \in \mathbb{R}^{n \times n}$ is a prescribed nonsingular matrix. Let $A=W+i T \in \mathbb{C}^{n \times n}$ be defined in (2), $\alpha$ a nonnegative constant, and $\beta$ a positive constant. Then the iteration matrix $M_{\alpha, \beta}$ of GPMHSS method is

$$
\begin{aligned}
M_{\alpha, \beta}= & (\beta V+W+T)^{-1}(\beta V+i(W-T)) \\
& \times(\alpha V+W-T)^{-1}(\alpha V-i(W+T)),
\end{aligned}
$$

which satisfies

$$
\rho\left(M_{\alpha, \beta}\right) \leq \max _{\lambda_{i} \in \lambda(\widehat{W})} \frac{\sqrt{\beta^{2}+\lambda_{i}^{2}}}{\alpha+\lambda_{i}} \max _{\mu_{i} \in \mu(\widehat{T})} \frac{\sqrt{\alpha^{2}+\mu_{i}^{2}}}{\beta+\mu_{i}},
$$

where $\lambda(\widehat{W})$ and $\mu(\widehat{T})$, respectively, are the spectral sets of the matrices $\widehat{W}$ and $\widehat{T}$.

In addition, let $\mu_{\min }$ and $\lambda_{\min }$, respectively, be the smallest eigenvalues of the matrices $\widehat{W}$ and $\widehat{T}$. If

$$
\sqrt{\alpha^{2}+\mu_{\min }^{2}}-\mu_{\min } \leq \beta<\sqrt{\alpha^{2}+2 \alpha \lambda_{\min }}
$$

then the DGPMHSS iteration (5) converges to the unique solution of the linear system (2).

Proof. Let

$$
\begin{array}{ll}
M_{1}=\alpha V+W-T, & N_{1}=\alpha V-i(W+T), \\
M_{2}=\beta V+W+T, & N_{2}=\beta V+i(W-T) .
\end{array}
$$


Obviously, $\alpha V+W-T$ and $\beta V+W+T$ are nonsingular for any nonnegative constants $\alpha$ or positive constants $\beta$. So formula (8) is valid.

The iteration matrix $M_{\alpha, \beta}$ is similar to

$$
\begin{aligned}
\widehat{M}_{\alpha, \beta}= & (\beta V+i(W-T))(\alpha V+W-T)^{-1} \\
& \times(\alpha V-i(W+T))(\beta V+W+T)^{-1} .
\end{aligned}
$$

That is,

$$
\begin{aligned}
\widehat{M}_{\alpha, \beta}= & R^{T}\left(\beta I+i R^{-T}(W-T) R^{-1}\right) \\
& \times R R^{-1}\left(\alpha I+R^{-T}(W-T) R^{-1}\right)^{-1} R^{-T} \\
& \times R^{T}\left(\alpha I-i R^{-T}(W+T) R^{-1}\right) \\
& \times R R^{-1}\left(\beta I+R^{-T}(W+T) R^{-1}\right)^{-1} R^{-T} \\
= & R^{T}\left(\beta I+i R^{-T}(W-T) R^{-1}\right) \\
& \times\left(\alpha I+R^{-T}(W-T) R^{-1}\right)^{-1} \\
& \times\left(\alpha I-i R^{-T}(W+T) R^{-1}\right) \\
& \times\left(\beta I+R^{-T}(W+T) R^{-1}\right)^{-1} R^{-T} \\
= & R^{T}(\beta I+i \widehat{W})(\alpha I+\widehat{W})^{-1} \\
& \times(\alpha I-i \widehat{T})(\beta I+\widehat{T})^{-1} R^{-T} .
\end{aligned}
$$

Therefore, we have

$$
\begin{aligned}
\rho( & \left.M_{\alpha, \beta}\right) \\
= & \rho\left(\widehat{M}_{\alpha, \beta}\right) \\
= & \rho\left(R^{T}(\beta I+i \widehat{W})(\alpha I+\widehat{W})^{-1}\right. \\
& \left.\quad \times(\alpha I-i \widehat{T})(\beta I+\widehat{T})^{-1} R^{-T}\right) \\
= & \rho\left((\beta I+i \widehat{W})(\alpha I+\widehat{W})^{-1}(\alpha I-i \widehat{T})(\beta I+\widehat{T})^{-1}\right) \\
\leq & \left\|(\beta I+i \widehat{W})(\alpha I+\widehat{W})^{-1}\right\|_{2} \\
& \times\left\|(\alpha I-i \widehat{T})(\beta I+\widehat{T})^{-1}\right\|_{2}
\end{aligned}
$$

Since $W \in \mathbb{R}^{n \times n}$ and $T \in \mathbb{R}^{n \times n}$, respectively, are symmetric positive definite and symmetric positive semidefinite and $R$ is a nonsingular matrix, $\widehat{W}$ and $\widehat{T}$ are symmetric positive definite and symmetric positive semidefinite, respectively. Therefore, there exist orthogonal matrices $S_{1}, S_{2} \in \mathbb{R}^{n \times n}$ such that

$$
S_{1}^{T} \widehat{W} S_{1}=\Lambda_{\widehat{W}}, \quad S_{2}^{T} \widehat{T} S_{2}=\Lambda_{\widehat{T}}
$$

where $\Lambda_{\widehat{W}}=\operatorname{diag}\left(\lambda_{1}, \lambda_{2}, \ldots, \lambda_{n}\right)$ and $\Lambda_{\widehat{T}}=$ $\operatorname{diag}\left(\mu_{1}, \mu_{2}, \ldots, \mu_{n}\right)$ with $\lambda_{i}>0(1 \leq i \leq n)$ and $\mu_{i} \geq 0(1 \leq i \leq n)$ being the eigenvalues of the matrices $\widehat{W}$ and $\widehat{T}$, respectively.

Through simple calculations, we can get that

$$
\begin{aligned}
\rho\left(M_{\alpha, \beta}\right) & \leq \max _{\lambda_{i} \in \lambda(\widehat{W})}\left|\frac{\beta+i \lambda_{i}}{\alpha+\lambda_{i}}\right| \max _{\mu_{i} \in \mu(\widehat{T})}\left|\frac{\alpha-i \mu_{i}}{\beta+\mu_{i}}\right| \\
& =\max _{\lambda_{i} \in \lambda(\widehat{W})} \frac{\sqrt{\beta^{2}+\lambda_{i}^{2}}}{\alpha+\lambda_{i}} \max _{\mu_{i} \in \mu(\widehat{T})} \frac{\sqrt{\alpha^{2}+\mu_{i}^{2}}}{\beta+\mu_{i}},
\end{aligned}
$$

which gives the upper bound for $\rho\left(M_{\alpha, \beta}\right)$ in (9). The next proof is similar to that of Theorem 1 in [17]. Here it is omitted.

The approach to minimize the upper bound is very important in theoretical viewpoint. However, it is not practical since the corresponding spectral radius of the iteration matrix $M_{\alpha, \beta}$ in (9) is not optimal. How to choose the suitable preconditioners and parameters for practical problem is still a great challenge.

\section{The IDGPMHSS Iteration}

In the DGPMHSS method, it is required to solve two systems of linear equations whose coefficient matrices are $\alpha V+W-T$ and $\beta V+W+T$, respectively. However, this may be very costly and impractical in actual implementation. To overcome this disadvantage and improve the computational efficiency of the DGPMHSS iteration method, we propose to solve the two subproblems iteratively $[12,18]$, which leads to IDGPMHSS iteration scheme. Its convergence can be shown in a similar way to that of the IHSS iteration method, using Theorem 3.1 of [12]. Since $\alpha V+W-T$ and $\beta V+W+T$ are symmetric positive definite, some Krylov subspace methods (such as CG) can be employed to gain its solution easily. Of course, if good preconditioners for matrices $\alpha V+W-T$ and $\beta V+W+T$ are available, we can use the preconditioned conjugate gradient (PCG) method instead of CG for the two inner systems that yields a better performance of IDGPMHSS method. If either $\alpha V+W-T$ or $\beta V+W+T$ (or both) is Toeplitz, we can use fast algorithms for solution of the corresponding subsystems [19].

\section{Numerical Examples}

In this section, we give some numerical examples to demonstrate the performance of the DGPMHSS and IDGPMHSS methods for solving the linear system (2). Numerical comparisons with the GPMHSS method are also presented to show the advantage of the DGPMHSS method.

In our implementation, the initial guess is chosen to be $x^{(0)}=0$ and the stopping criteria for outer iterations is

$$
\frac{\left\|b-A x^{(k)}\right\|_{2}}{\|b\|_{2}} \leq 10^{-6} .
$$

The preconditioner $V$ used in GPMHSS method is chosen to be $V=W-T$. For the sake of comparing, the corresponding 
TABLE 1: The experimentally optimal parameters and the corresponding spectral radii for the iteration matrices of GPMHSS and DGPMHSS with $V=W-T$ and $m=8$.

\begin{tabular}{ccccc}
\hline & \multicolumn{5}{c}{$\sigma_{2}$} \\
& 10 & 50 & 80 & 100 \\
\hline GPMHSS & & & & \\
$\alpha_{G}^{*}$ & 1.1 & 1.5 & 2.2 & 2 \\
$\rho\left(M_{\alpha_{G}^{*}}\right)$ & 0.5009 & 0.5222 & 0.5667 & 0.6274 \\
DGPMHSS & & & & \\
$\alpha_{D}^{*}$ & 1.1 & 1.5 & 2.2 & 2 \\
$\beta_{D}^{*}$ & 1 & 0.9 & 0.8 & 0.8 \\
$\rho\left(M_{\alpha_{D}^{*}, \beta_{D}^{*}}\right)$ & 0.5001 & 0.4986 & 0.5012 & 0.4901 \\
\hline
\end{tabular}

preconditioner $V$ used in DGPMHSS method is chosen to be $V=W-T$. Since the numerical results in $[2,3]$ show that the PMHSS iteration method outperforms the MHSS and HSS iteration methods when they are employed as preconditioners for the GMRES method or its restarted variants [20], we just examine the efficiency of DGPMHSS iteration method as a solver for solving complex symmetric linear system (2) by comparing the iteration numbers (denoted as IT) and CPU times (in seconds, denoted as CPU(s)) of DGPMHSS method with GPMHSS method.

Example 3 (see [5, 21-23]). Consider the following form of the Helmholtz equation:

$$
-\Delta u+\sigma_{1} u+i \sigma_{2} u=f
$$

where $\sigma_{1}, \sigma_{2}$ are real coefficient functions and $u$ satisfies Dirichlet boundary conditions in $D=[0,1] \times[0,1]$. The above equation describes the propagation of damped time harmonic waves. We take $H$ to be the five-point centered difference matrix approximating the negative Laplacian operator on a uniform mesh with mesh size $h=1 /(m+1)$. The matrix $H \in \mathbb{R}^{n \times n}$ possesses the tensor-product form $H=B_{m} \otimes I+I \otimes B_{m}$ with $B_{m}=h^{-2} \cdot \operatorname{tridiag}(-1,2,-1) \in \mathbb{R}^{m \times m}$. Hence, $H$ is an $n \times n$ block-tridiagonal matrix, with $n=m^{2}$. This leads to the complex symmetric linear system (2) of the form

$$
\left[\left(H+\sigma_{1} I\right)+i \sigma_{2} I\right] x=b
$$

In addition, we set $\sigma_{1}=100$ and the right-hand side vector $b$ to be $b=(1+i) A \mathbf{1}$, with $\mathbf{1}$ being the vector of all entries equal to 1 . As before, we normalize the system by multiplying both sides by $h^{2}$.

As is known, the spectral radius of the iteration matrix may be decisive for the convergence of the iteration method. The spectral radius corresponding to the iteration method is necessary to consider. The comparisons of the spectral radius of the two different iteration matrices derived by GPMHSS and DGPMHSS methods with different mesh size are performed in Tables 1, 2, 3, and 4. In Tables 1-4, we used the optimal values of the parameters $\alpha$ and $\beta$, denoted by $\alpha_{G}^{*}$
TABLE 2: The experimentally optimal parameters and the corresponding spectral radii for the iteration matrices of GPMHSS and DGPMHSS with $V=W-T$ and $m=16$.

\begin{tabular}{ccccc}
\hline & \multicolumn{3}{c}{$\sigma_{2}$} & \\
& 10 & 50 & 80 & 100 \\
\hline GPMHSS & & & & \\
$\alpha_{G}^{*}$ & 1.1 & 1.5 & 2.2 & 1.8 \\
$\rho\left(M_{\alpha_{G}^{*}}\right)$ & 0.5010 & 0.5230 & 0.5697 & 0.6337 \\
DGPMHSS & & & & \\
$\alpha_{D}^{*}$ & 1.1 & 1.5 & 2.2 & 1.8 \\
$\beta_{D}^{*}$ & 1 & 1 & 0.9 & 0.9 \\
$\rho\left(M_{\alpha_{D}^{*}, \beta_{D}^{*}}\right)$ & 0.5004 & 0.5063 & 0.5235 & 0.5097 \\
\hline
\end{tabular}

TABLE 3: The experimentally optimal parameters and the corresponding spectral radii for the iteration matrices of GPMHSS and DGPMHSS with $V=W-T$ and $m=24$.

\begin{tabular}{ccccc}
\hline & & \multicolumn{3}{c}{$\sigma_{2}$} \\
& 10 & 50 & 80 & 100 \\
\hline GPMHSS & & & & \\
$\alpha_{G}^{*}$ & 1.1 & 1.5 & 2.2 & 1.6 \\
$\rho\left(M_{\alpha_{G}^{*}}\right)$ & 0.5011 & 0.5234 & 0.5708 & 0.6360 \\
DGPMHSS & & & & \\
$\alpha_{D}^{*}$ & 1.1 & 1.5 & 2.2 & 1.8 \\
$\beta_{D}^{*}$ & 1 & 1 & 1 & 1 \\
$\rho\left(M_{\alpha_{D}^{*}, \beta_{D}^{*}}\right)$ & 0.5005 & 0.5081 & 0.5289 & 0.5091 \\
\hline
\end{tabular}

TABLE 4: The experimentally optimal parameters and the corresponding spectral radii for the iteration matrices of GPMHSS and DGPMHSS with $V=W-T$ and $m=32$.

\begin{tabular}{ccccc}
\hline & & \multicolumn{3}{c}{$\sigma_{2}$} \\
\hline GPMHSS & 10 & 50 & 80 & 100 \\
$\alpha_{G}^{*}$ & 1.1 & 1.5 & 2.2 & 1.7 \\
$\rho\left(M_{\alpha_{G}^{*}}\right)$ & 0.5011 & 0.5235 & 0.5714 & 0.6372 \\
DGPMHSS & & & & \\
$\alpha_{D}^{*}$ & 1.1 & 1.5 & 2.2 & 1.8 \\
$\beta_{D}^{*}$ & 1 & 1 & 1 & 1 \\
$\rho\left(M_{\alpha_{D}^{*}, \beta_{D}^{*}}\right)$ & 0.5005 & 0.5089 & 0.5310 & 0.5139 \\
\hline
\end{tabular}

for GPMHSS method, and $\alpha_{D}^{*}, \beta_{D}^{*}$ for DGPMHSS method. These parameters are obtained experimentally with the least spectral radius for the iteration matrices of the two methods.

From Tables 1-4, one can see that with the mesh size creasing, the trend of the experimentally optimal parameter of the GPMHSS and DGPMHSS methods is relatively stable. In Tables 1-4, we observe that the optimal spectral radius of DGPMHSS method is still smaller than those of the GPMHSS method, which implies that the DGPMHSS method may outperform the GPMHSS method. To this end, we need 
TABLE 5: RES, CPU(s), and IT for PMHSS and GPMHSS with $V=$ $W-T$ and $m=8$.

\begin{tabular}{lcccc}
\hline & \multicolumn{5}{c}{$\sigma_{2}$} \\
& 10 & 50 & 80 & 100 \\
\hline GPMHSS & & & & \\
RES & $9.818 e-7$ & $9.8917 e-7$ & $9.1469 e-7$ & $6.306 e-7$ \\
CPU(s) & 0.015 & 0.016 & 0.016 & 0.016 \\
IT & 20 & 21 & 24 & 30 \\
DGPMHSS & & & & \\
RES & $9.0916 e-7$ & $7.0211 e-7$ & $4.9072 e-7$ & $6.5183 e-7$ \\
CPU(s) & 0.015 & 0.015 & 0.015 & 0.013 \\
IT & 20 & 19 & 18 & 17 \\
\hline
\end{tabular}

TABLE 6: RES, CPU(s), and IT for PMHSS and GPMHSS with $V=$ $W-T$ and $m=16$.

\begin{tabular}{lcccc}
\hline \multicolumn{5}{c}{$\sigma_{2}$} \\
& 10 & 50 & 80 & 100 \\
\hline GPMHSS & & & & \\
RES & $9.8506 e-7$ & $9.4015 e-7$ & $8.9127 e-7$ & $9.4832 e-7$ \\
CPU(s) & 0.031 & 0.046 & 0.046 & 0.063 \\
IT & 20 & 21 & 24 & 29 \\
DGPMHSS & & & & \\
RES & $9.3425 e-7$ & $7.2018 e-7$ & $7.4847 e-7$ & $8.9905 e-7$ \\
CPU(s) & 0.031 & 0.032 & 0.031 & 0.031 \\
IT & 20 & 20 & 20 & 19 \\
\hline
\end{tabular}

TABLE 7: RES, CPU(s), and IT for PMHSS and GPMHSS with $V=$ $W-T$ and $m=24$

\begin{tabular}{lcccc}
\hline & \multicolumn{5}{c}{$\sigma_{2}$} \\
& 10 & 50 & 80 & 100 \\
\hline GPMHSS & & & & \\
RES & $9.8907 e-7$ & $9.6059 e-7$ & $9.7633 e-7$ & $7.5427 e-7$ \\
CPU(s) & 0.094 & 0.094 & 0.11 & 0.125 \\
IT & 20 & 21 & 24 & 29 \\
DGPMHSS & & & & \\
RES & $9.5055 e-7$ & $9.5856 e-7$ & $7.8461 e-7$ & $7.6081 e-7$ \\
CPU(s) & 0.078 & 0.078 & 0.094 & 0.094 \\
IT & 20 & 20 & 21 & 20 \\
\hline
\end{tabular}

to examine the efficiency of the GPMHSS and DGPMHSS methods for solving the systems of linear equations $A x=b$, where $A$ is described.

In Tables 5, 6, 7, and 8, we list the numbers of iteration steps and the computational times for GPMHSS and DGPMHSS iteration methods using the optimal parameters in Tables 1-4. In Tables 5-8, "RES" denotes the relative residual error.

From Tables 5-8, we see that the DGPMHSS method is the best among the two methods in terms of number of iteration steps and computational time. For the GPMHSS and DGPMHSS methods, the CPU's time grows with the problem size whereas the presented results in Tables 5-8 show that in all cases the DGPMHSS method is superior to the GPMHSS
TABLE 8: RES, CPU(s), and IT for PMHSS and GPMHSS with $V=$ $W-T$ and $m=32$.

\begin{tabular}{lcccc}
\hline & \multicolumn{5}{c}{$\sigma_{2}$} \\
& 10 & 50 & 80 & 100 \\
\hline GPMHSS & & & & \\
RES & $9.9181 e-7$ & $9.8976 e-7$ & $6.0829 e-7$ & $9.1273 e-7$ \\
CPU(s) & 0.172 & 0.172 & 0.203 & 0.235 \\
IT & 20 & 21 & 25 & 28 \\
DGPMHSS & & & & \\
RES & $9.5956 e-7$ & $5.5697 e-7$ & $5.6167 e-7$ & $5.5736 e-7$ \\
CPU(s) & 0.156 & 0.156 & 0.187 & 0.172 \\
IT & 20 & 20 & 22 & 21 \\
\hline
\end{tabular}

method. That is to say, under certain conditions, compared with the GPMHSS method, the DGPMHSS method may be given the priority for solving the complex symmetric linear system $(W+i T) x=b$ with $-W \preceq T \prec W$.

As already noted, in the two-half steps of the DGPMHSS iteration, there is a need to solve two systems of linear equations, whose coefficient matrices are $\alpha V+W-T$ and $\beta V+$ $W+T$, respectively. This can be very costly and impractical in actual implementation. We use the IDGPMHSS method to solve the systems of linear equations (2) in the actual implementation. That is, it is necessary to solve two systems of linear equations with $\alpha V+W-T$ and $\beta V+W+T$ by using the IDGPMHSS iteration. It is easy to know that $\alpha V+W-T$ and $\beta V+W+T$ are symmetric and positive definite. So, solving the above two subsystems, the CG method can be employed.

In our computations, the inner CG iteration is terminated if the current residual of the inner iterations satisfies

$$
\frac{\left\|p^{(j)}\right\|_{2}}{\left\|r^{(k)}\right\|_{2}} \leq 0.1 \tau^{(k)}, \quad \frac{\left\|q^{(j)}\right\|_{2}}{\left\|r^{(k)}\right\|_{2}} \leq 0.1 \tau^{(k)},
$$

where $p^{(j)}$ and $q^{(j)}$ are, respectively, the residuals of the $j$ th inner CG for $\alpha V+W-T$ and $\beta V+W+T . r^{(k)}$ is the $k$ th outer IDGPMHSS iteration; $\tau$ is a tolerance.

Some results are listed in Tables 9 and 10 for $m=24$ and 32, which are the numbers of outer IDGPMHSS iteration (it.s), the average numbers (avg1) of inner CG iteration for $\alpha V+W-T$ and the average numbers (avg2) of CG iteration for $\beta V+W+T$.

In our numerical computations, it is easy to find the fact that the choice of $\tau$ is important to the convergence rate of the IDGPMHSS method. According to Tables 9 and 10, the iteration numbers of the IDGPMHSS method generally increase when $\tau$ decreases. Meanwhile, the iteration numbers of the IDGPMHSS method generally increase when $\sigma_{2}$ increases.

\section{Conclusion}

In this paper, we have generalized the GPMHSS method to the DGPMHSS method for a class of complex symmetric linear systems $(W+i T) x=b$ with real symmetric matrices $W, T \in \mathbb{R}^{n \times n}$ satisfying $-W \preceq T \prec W$ under certain conditions. Theoretical analysis shows that for any initial guess 
TABLE 9: Convergence results for the IDGPMHSS iteration with $m=24$.

\begin{tabular}{lcccccccccc}
\hline$\left(\alpha_{G}^{*}, \beta_{G}^{*}\right)$ & $\sigma_{2}$ & it.s & $\begin{array}{c}\tau=0.95 \\
\text { avg1 }\end{array}$ & avg2 & it.s & avg1 & avg2 & it.s & avg1 & avg2 \\
\hline$(1.1,1)$ & 10 & 51 & 20.9 & 18.2 & 68 & 26.1 & 23.7 & 84 & 30.4 & 28.3 \\
$(1.5,1)$ & 50 & 54 & 23.5 & 18.3 & 70 & 28.0 & 23.9 & 86 & 33.0 & 28.4 \\
$(2.2,1)$ & 80 & 57 & 25.0 & 20.1 & 72 & 30.0 & 25.3 & 88 & 34.2 & 30.0 \\
$(1.8,1)$ & 100 & 58 & 27.5 & 18.9 & 74 & 31.9 & 24.3 & 89 & 36.1 & 28.7 \\
\hline
\end{tabular}

TABLE 10: Convergence results for the IDGPMHSS iteration with $m=32$.

\begin{tabular}{lcccccccccc}
\hline$\left(\alpha_{G}^{*}, \beta_{G}^{*}\right)\left(\alpha_{G}^{*}, \beta_{G}^{*}\right)$ & $\sigma_{2}$ & it.s & avg1 & avg2 & it.s & avg1 & avg2 & it.s & $\begin{array}{c}\tau=0.85 \\
\text { avg1 }\end{array}$ \\
\hline$(1.1,1)$ & 10 & 66 & 27.1 & 23.6 & 90 & 34.2 & 31.1 & 111 & 40.0 \\
$(1.5,1)$ & 50 & 70 & 30.6 & 23.7 & 93 & 37.5 & 31.3 & 114 & 43.4 \\
$(2.2,1)$ & 80 & 75 & 35.0 & 24.2 & 97 & 41.1 & 31.8 & 118 & 47.0 & 37.4 \\
$(1.8,1)$ & 100 & 77 & 36.1 & 25.3 & 98 & 42.2 & 32.4 & 119 & 48.0 & 38.3 \\
\hline
\end{tabular}

the DGPMHSS method converges to the unique solution of the linear system for a wide range of the parameters. Then, an inexact version has been presented and implemented for saving the computational cost. Numerical experiments show that DGPMHSS method and IDGPMHSS method are efficient and competitive.

\section{Conflict of Interests}

The authors declare that there is no conflict of interests regarding the publication of this paper.

\section{Acknowledgments}

The authors would like to thank the anonymous referees for their helpful suggestions, which greatly improved the paper. The authors would like to thank Yan-Jun Liang, Ting Wang, Ming-Yue Shao, and Man-Man Wang for helpful discussions. This work is also supported by the National College Students' Innovative and Entrepreneurial Training Program (201310479069). This research was supported by NSFC (no. 11301009), Science and Technology Development Plan of Henan Province (no. 122300410316), and Natural Science Foundations of Henan Province (no. 13A110022).

\section{References}

[1] A. Feriani, F. Perotti, and V. Simoncini, "Iterative system solvers for the frequency analysis of linear mechanical systems," Computer Methods in Applied Mechanics and Engineering, vol. 190, pp. 1719-1739, 2000.

[2] Z.-Z. Bai, M. Benzi, and F. Chen, "Modified HSS iteration methods for a class of complex symmetric linear systems," Computing, vol. 87, no. 3-4, pp. 93-111, 2010.

[3] Z.-Z. Bai, M. Benzi, and F. Chen, "On preconditioned MHSS iteration methods for complex symmetric linear systems," Numerical Algorithms, vol. 56, no. 2, pp. 297-317, 2011.

[4] S. R. Arridge, "Optical tomography in medical imaging," Inverse Problems, vol. 15, no. 2, pp. R41-R93, 1999.
[5] D. Bertaccini, "Efficient preconditioning for sequences of parametric complex symmetric linear systems," Electronic Transactions on Numerical Analysis, vol. 18, pp. 49-64, 2004.

[6] A. Frommer, T. Lippert, B. Medeke, and K. Schilling, Numerical Challenges in Lattice Quantum Chromodynamics, vol. 15 of Lecture notes in computational science and engineering, Springer, Heidelberg, Germany, 2000.

[7] D. Day and M. A. Heroux, "Solving complex-valued linear systems via equivalent real formulations," SIAM Journal on Scientific Computing, vol. 23, no. 2, pp. 480-498, 2001.

[8] Z.-Z. Bai, "Block alternating splitting implicit iteration methods for saddle-point problems from time-harmonic eddy current models," Numerical Linear Algebra with Applications, vol. 19, no. 6, pp. 914-936, 2012.

[9] T. Sogabe and S.-L. Zhang, "A COCR method for solving complex symmetric linear systems," Journal of Computational and Applied Mathematics, vol. 199, no. 2, pp. 297-303, 2007.

[10] M. Benzi and D. Bertaccini, "Block preconditioning of realvalued iterative algorithms for complex linear systems," IMA Journal of Numerical Analysis, vol. 28, no. 3, pp. 598-618, 2008.

[11] Z.-Z. Bai, "Structured preconditioners for nonsingular matrices of block two-by-two structures," Mathematics of Computation, vol. 75, no. 254, pp. 791-815, 2006.

[12] Z.-Z. Bai, G. H. Golub, and M. K. Ng, "Hermitian and skew-Hermitian splitting methods for non-Hermitian positive definite linear systems," SIAM Journal on Matrix Analysis and Applications, vol. 24, no. 3, pp. 603-626, 2003.

[13] Z.-Z. Bai, M. Benzi, F. Chen, and Z.-Q. Wang, "Preconditioned MHSS iteration methods for a class of block two-by-two linear systems with applications to distributed control problems," IMA Journal of Numerical Analysis, vol. 33, no. 1, pp. 343-369, 2013.

[14] W.-W. Xu, "A generalization of preconditioned MHSS iteration method for complex symmetric indefinite linear systems," Applied Mathematics and Computation, vol. 219, no. 21, pp. 10510-10517, 2013.

[15] L. Li, T.-Z. Huang, and X.-P. Liu, "Asymmetric Hermitian and skew-Hermitian splitting methods for positive definite linear systems," Computers \& Mathematics with Applications, vol. 54, no. 1, pp. 147-159, 2007. 
[16] A.-L. Yang, J. An, and Y.-J. Wu, "A generalized preconditioned HSS method for non-Hermitian positive definite linear systems," Applied Mathematics and Computation, vol. 216, no. 6, pp. 1715-1722, 2010.

[17] M. Dehghana, M. D. Madiseha, and M. Hajarian, "A GPMHSS method for a class of complex symmetric linear systems," Mathematical Modelling and Analysis, vol. 18, no. 4, pp. 561-576, 2013.

[18] Z.-Z. Bai, G. H. Golub, and M. K. Ng, "On inexact Hermitian and skew-Hermitian splitting methods for non-Hermitian positive definite linear systems," Linear Algebra and its Applications, vol. 428, no. 2-3, pp. 413-440, 2008.

[19] R. H. Chan and M. K. Ng, "Conjugate gradient methods for Toeplitz systems," SIAM Review, vol. 38, no. 3, pp. 427-482, 1996.

[20] Y. Saad, Iterative Methods for Sparse Linear Systems, PWS Publishing Company, Boston, Mass, USA, 1996.

[21] R. W. Freund, "Conjugate gradient-type methods for linear systems with complex symmetric coefficient matrices," SIAM Journal on Scientific and Statistical Computing, vol. 13, no. 1, pp. 425-448, 1992.

[22] X. Li, A. L. Yang, and Y. J. Wu, "Lopsided PMHSS iteration method for a class of complex symmetric linear systems," Numerical Algorithms, 2013.

[23] S.-L. Wu, T.-Z. Huang, L. Li, and L.-L. Xiong, "Positive stable preconditioners for symmetric indefinite linear systems arising from Helmholtz equations," Physics Letters A, vol. 373, no. 29, pp. 2401-2407, 2009. 


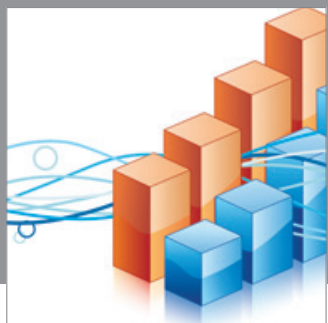

Advances in

Operations Research

mansans

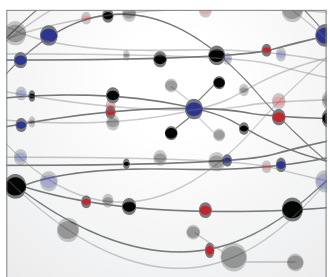

The Scientific World Journal
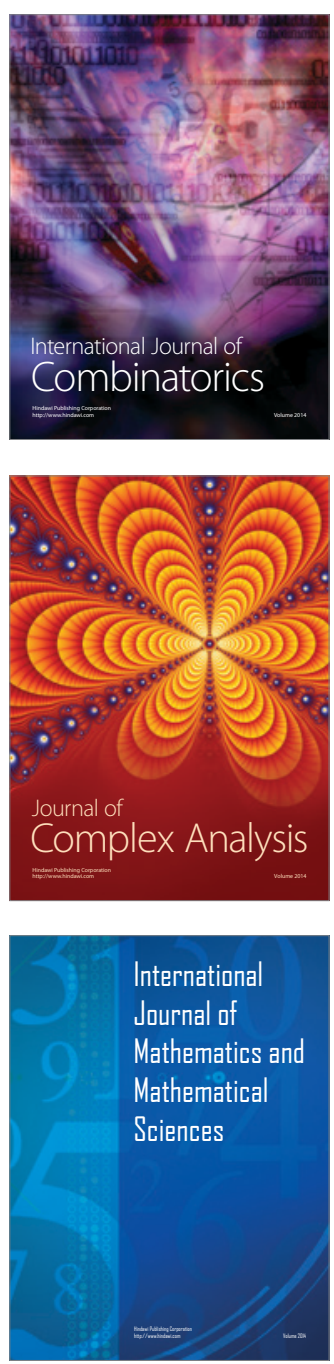
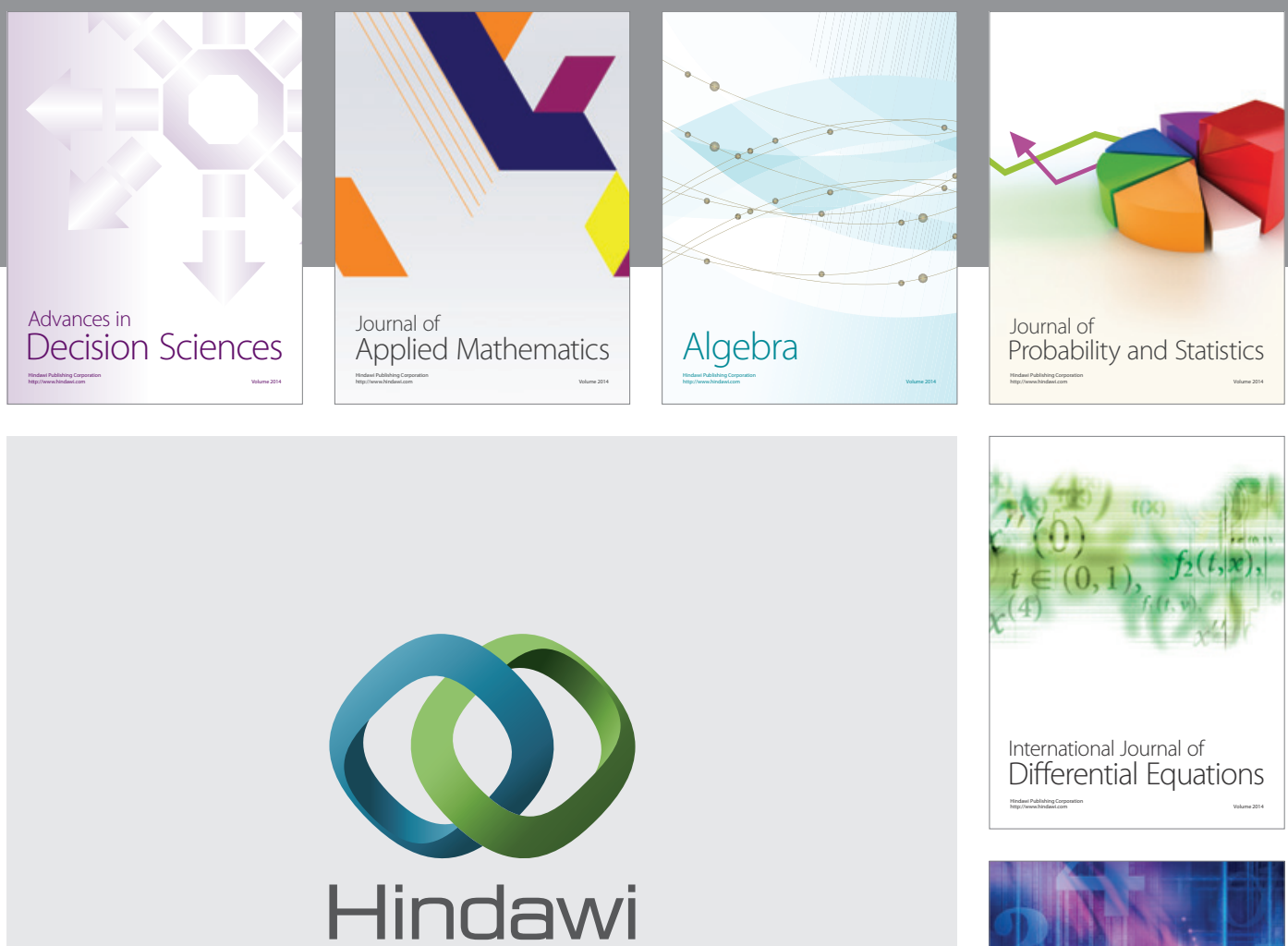

Submit your manuscripts at http://www.hindawi.com
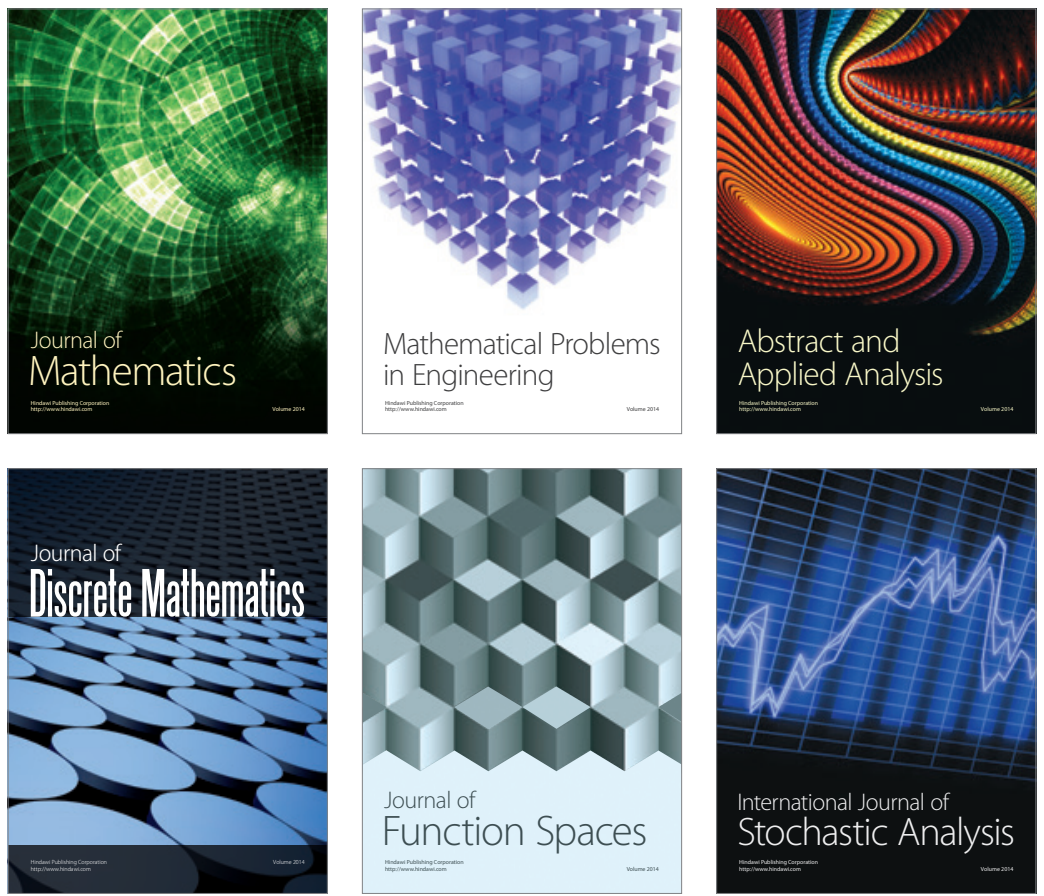

Journal of

Function Spaces

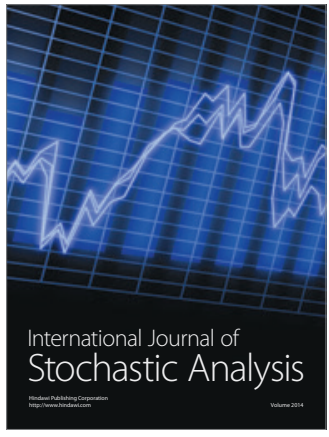

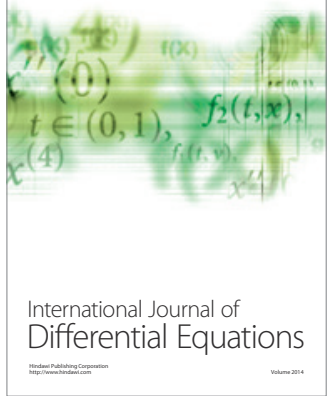
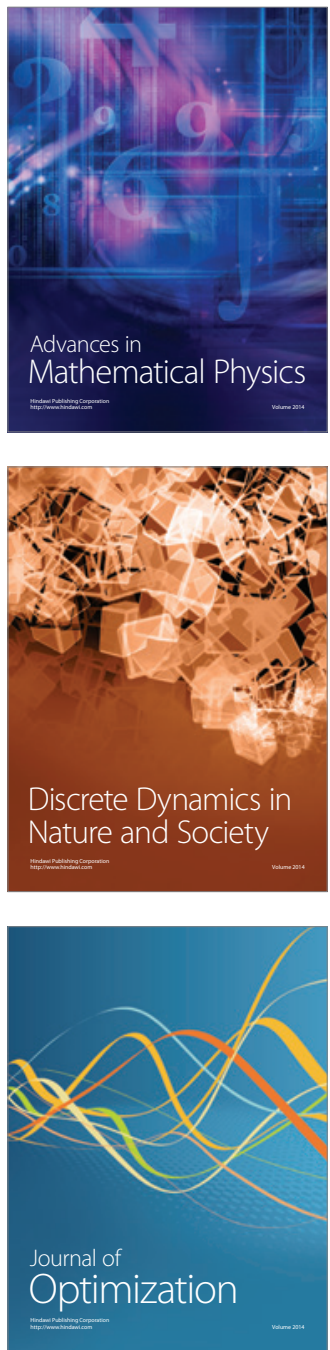